\title{
WHO-Empfehlung für Kinder auf dem Prüfstand
}

\author{
Die Weltgesundheitsorganisation (WHO) empfiehlt eine \\ präventive Isoniazid-Therapie (IPT) für Kleinkinder, die engen \\ Kontakt zu Personen mit infektiöser Tuberkulose (TB) haben. \\ A. M. Mandalakas et al. haben ermittelt, wie kosteneffektiv \\ diese päventive Maßnahme bei hohem Ansteckungsrisiko ist. \\ Thorax 2013; 68: 247-255
}

Die Wissenschaftler entwickelten hierzu ein Entscheidungsbaummodell, um die Ergebnisse von 5 TB-Screening-Strategien bei Vorschulkindern mit Kontakt zu TB-Erkrankten im eigenen Haushalt zu überprüfen. Eine Strategie sah dabei keine Testung vor, sondern ausschließlich die Gabe von IPT basierend auf der Risikoabschätzung nach Alter und Exposition. Andere Strategien umfassten die TB-Testung, entweder mit einem Tuberkulin-Hauttest (TST), Interferon-gamma-Freisetzungstest (IGRA) oder einer Kombination aus beiden. Da sich das Infektionsrisiko für Kinder mit dem Al- ter verändert, nahmen die Autoren Modellrechnungen für die Altersgruppen 0-2 Jahre und 3-5 Jahre separat vor. Bei der Einnahme der IPT wurde von einer realistisch geringen Adhärenz ausgegangen.

In der Modellkohorte der 0- bis 2-Jährigen erwies sich die reine IPT-Strategie ohne vorherige Testung als kosteneffektiver als alle anderen. In der Kohorte der 3- bis 5-Jährigen war die Strategie eines TST gefolgt von IGRA bei positivem TST am kosteneffektivsten. Allerdings war die Kostensteigerung in Abhängigkeit von der Rate der Infektionen mit M. tuberculosis bedeutsam, sodass in Regionen mit stark begrenzten Ressourcen auch in dieser Altersgruppe die IPT-Strategie ohne Test zu bevorzugen ist. Eine Steigerung der Kosteneffektivität der Tuberkuloseprävention in Hochrisikopopulationen könnte durch eine verbesserte Analyse der Kontakte der Indexpatienten, einen vermehrten Einsatz der IPT und eine erhöhte Compliance bei der IPT erreicht werden.

\section{Fazit}

Das Tuberkulose-Screening im Sinne der Umfeldanalyse von Indexpatienten und die Durchführung der IPT stellen zusammen eine hochgradig kosteneffektive Intervention bei Kleinkindern dar. Dabei ist die von der WHO empfohlene IPT ohne vorherige Testung nach den von den Autoren durchgeführten Modellrechnungen die kosteneffektivste Maßnahme bei 0 - bis 2-Jährigen und die nach Meinung der Autoren zu bevorzugende Strategie bei 3- bis 5-Jährigen. Sie betonen, dass in keinem Fall auf die IPT verzichtet werden sollte, bloß weil keine Testkapazitäten vorhanden sind.

\section{Friederike Klein, München}

